\title{
An Assessment of the Fe XVIII and Fe XIX Line Ratios from the Chandra Grating Observations of Capella
}

\author{
P. Desai, N. S. Brickhouse, J. J. Drake, A. K. Dupree, R. J. Edgar, R. Hoogerwerf, \\ V. Kashyap, and B. J. Wargelin \\ Harvard-Smithsonian Center for Astrophysics, 60 Garden Street, Cambridge, MA 02138 \\ pdesai@cfa.harvard.edu, nbrickhouse@cfa.harvard.edu, jdrake@cfa.harvard.edu, \\ adupree@cfa.harvard.edu, redgar@cfa.harvard.edu, \\ rhoogerwerf@cfa.harvard.edu, vkashyap@cfa.harvard.edu, \\ bwargelin@cfa.harvard.edu \\ R. K. Smith \\ NASA Goddard Space Flight Center/JHU, Code 662, Greenbelt, MD 20771 \\ rsmith@milkyway.gsfc.nasa.gov \\ D. P. Huenemoerder \\ MIT Center for Space Research, 70 Vassar Street, Cambridge, MA 02139 \\ dph@space.mit.edu \\ D. A. Liedahl \\ Lawrence Livermore National Laboratory, P.O. Box 808, L-41, Livermore, CA 94551 \\ liedahl1@llnl.gov
}

ABSTRACT 
Observations of Fe XVIII and Fe XIX X-ray, EUV, and FUV line emission, formed at the peak of Capella's ( $\alpha$ Aurigae) emission measure distribution and ubiquitous in spectra of many cool stars and galaxies, provide a unique opportunity to test the robustness of Fe XVIII and Fe XIX spectral models. The Astrophysical Plasma Emission Code (APEC) is used to identify over 35 lines from these two ions alone, and to compare model predictions with spectra obtained with the Chandra Low Energy Transmission Grating and High Energy Transmission Grating Spectrometers, the Far Ultraviolet Spectroscopic Explorer, and the Extreme Ultraviolet Explorer. Some flux discrepancies larger than factors of two are found between observations of Fe XVIII and Fe XIX lines and predictions by APEC and other models in common usage. In particular the X-ray resonance lines for both ions are stronger than predicted by all models relative to the EUV resonance lines. The multiwavelength observations demonstrate the importance of including dielectronic recombination and proton impact excitation, and of using accurate wavelengths in spectral codes. These ions provide important diagnostic tools for $10^{7} \mathrm{~K}$ plasmas currently observed with Chandra, XMM-Newton, and FUSE.

Subject headings: atomic data — atomic processes — stars: individual (Capella) - ultraviolet: stars — X-rays: stars

\section{Introduction}

The Capella system (HD 34029, $\alpha$ Aurigae), consisting principally of two cool giant stars $(\mathrm{G} 8 \mathrm{III}+\mathrm{G} 1 \mathrm{III})$, is one of the strongest coronal X-ray sources, and offers an opportunity

to benchmark the models used in the interpretation of X-ray spectra from astrophysical plasmas. Although plasma codes have gone through major improvements (e.g., Mewe et al. 
1995; Brickhouse et al. 1995; Smith et al. 2001; Young et al. 2003), their accuracy and completeness for diagnostic analysis at high spectral resolution has yet to be fully assessed. Brickhouse \& Drake (2000) proposed the use of the Chandra grating calibration observations for a series of studies, collectively known as the Emission Line Project (ELP), to test spectral models of collisionally ionized plasmas. This paper compares current spectral models for Fe XVIII and Fe XIX with Chandra observations of Capella as a step towards ensuring that astrophysical interpretations of spectra are based on a sound understanding of the physical processes involved. The Capella spectrum is well studied, showing no evidence for flares (Brinkman et al. 2001; Canizares et al. 2000; Brickhouse et al. 2000). The emission measure distribution (EMD) of the Capella system shows a strong narrow peak at $6 \mathrm{MK}$, near the temperature of peak emissivity for Fe XVIII and Fe XIX, producing numerous strong transitions. Furthermore, line fluxes from these ions show only modest variability $(\sim 20 \%)$ over timescales of months to years (Dupree et al. 2005), validating the combined analysis of multiple observations with Chandra.

Although Fe L-shell (i.e. Fe XVII to Fe XXIV) X-ray lines offer powerful diagnostic potential for collisionally ionized plasmas, two long-standing atomic modeling problems originate from solar observations of neon-like Fe XVII (see Saba et al. 1999) that have only recently been addressed by laboratory programs (Brown et al. 1998; Laming et al. 2000; Beiersdorfer et al. 2002). Similar discrepancies between models and observations of other ions are now arising from Chandra spectra. For example, Xu et al. (2002) find that the observed Fe XVIII $3 s-2 p / 3 d-2 p$ ratio in the elliptical galaxy NGC 4636 is higher than predicted by APEC and similar to the ratio observed in Capella. The analogous Fe XVII ratio shows the same pattern of discrepancy, suggesting a common atomic physics origin. The ELP observations of Capella with three instruments offer a unique opportunity to compare models and observations over a broad spectral range. Comparisons among FUV, EUV, and X-ray lines of Fe XVIII and Fe XIX are particularly useful since the strong $\mathrm{n}=2 \rightarrow 2$ lines 
are essentially entirely produced by direct collisional excitation, and thus should be easier to interpret than FUV or X-ray lines, which can include contributions from other processes, such as proton impact excitation and dielectronic recombination (DR).

\section{Spectral Models}

We use the Astrophysical Plasma Emission Code version 1.3 (APEC, Smith et al. 2001) to predict the Capella spectrum. ${ }^{1}$ The APEC models for Fe XVIII and Fe XIX contain 501 and 994 fine-structure levels, respectively, up to principal quantum number $n=5$. They include the effective collision strengths and atomic transition probabilities calculated using the Hebrew University Lawrence Livermore Atomic Code (HULLAC, Liedahl et al. 1995). For Fe XVIII, the collision strengths for the $2 p^{5}{ }^{2} P_{1 / 2}-2 p^{5}{ }^{2} P_{3 / 2}$ transition include resonance excitation from R-matrix calculations (Berrington et al. 1998). Proton impact excitation rates within the ground state are included for Fe XVIII (Foster et al. 1994) and Fe XIX (R. Reid, private communication, 1999). Laboratory X-ray wavelengths (Brown et al. 2002) have been incorporated. APEC currently includes DR rates to excited levels of Fe XVII and H- and He-like ions, but not for the other Fe L-shell ions. Similarly, DR satellite lines are present in APEC for Fe XVII (Safranova et al 2001), but not for Fe XVIII and Fe XIX.

\footnotetext{
${ }^{1}$ APEC V1.3 models, calculated at the low density limit $\left(N_{e}=1.0 \mathrm{~cm}^{-3}\right)$, and the atomic rate data used to produce them are available at http://cxc.harvard.edu/atomdb/.
} 


\section{Data Analysis}

\subsection{Observations and Data Reduction}

Multiple spectra of Capella, acquired between 1999 August and 2002 October include pointings with the Chandra High Energy Transmission Grating (HETG) with the ACIS-S detector for a total exposure time of $182.2 \mathrm{ks}$, and with the Low Energy Transmission Grating (LETG) and HRC-S detector for a total exposure time of $234.2 \mathrm{ks}$. The HETG and LETG data, obtained from the Chandra archive, were reprocessed using CIAO version $3.0^{2}$ with only minor deviations from the standard pipeline procedures. Effective areas were generated for each dataset using the Chandra calibration database CALDB 2.8 and were exposure-time weighted to create average effective areas for the summed spectra.

Extreme Ultraviolet Explorer (EUVE) spectra obtained in 1999 September, which are nearly simultaneous with a Chandra LETG/HRC-S pointing, were processed using standard EUVE Guest Observer software (IRAF). Agreement between LETG and EUVE fluxes for the lines discussed in this paper is good to within about 5\%, and henceforth LETG fluxes will be used. The Far Ultraviolet Spectroscopic Explorer (FUSE) line fluxes are taken from spectra of Young et al. (2001).

\subsection{Modeling and Measurements}

We calculate the global continuum spectra produced by bremsstrahlung, radiative recombination continuum, and two-photon emission over the observed Chandra spectral range. We then fit the temperature of the continuum model to the line-free regions of the HETG spectrum, identified both from the APEC line list and by visual inspection, which yields a

\footnotetext{
${ }^{2}$ http://cxc.harvard.edu/ciao/
} 
temperature of $6 \mathrm{MK}$, near the peak of the EMD. Since the LETG spectrum is contaminated by high order emission, the same continuum model derived from the HETG data is also applied to the LETG fitting. We adopted the abundances of Brickhouse et al. (2000), who found no evidence for deviation from the solar abundances of Anders \& Grevesse (1989). Individual line fluxes from the Chandra spectra were measured using Sherpa (Freeman et al. 2001) to fit functions approximating the instrumental line profiles. Plus and minus orders were fit separately, with the requirement that the line fluxes be the same. A narrow range for the FWHM was allowed (for HETG, 0.01 - $0.0135 \AA$, and for LETG, 0.045 - $0.06 \AA$ ), standard binning was maintained, and the Cash statistic was applied (Cash 1979). Table 1 gives the observed fluxes for the Fe XVIII and Fe XIX lines with $1 \sigma$ errors.

\section{3. $\quad$ Model Assumptions}

A continuous EMD (Brickhouse et al. 2000), which is needed to estimate the contribution of line blends from ions over the entire temperature range, is normalized to the flux of the Fe XVIII $\lambda 93.92$ resonance line and used to predict the line fluxes given in Table 1 . We note that there is only a few percent difference between the single temperature continuum model and the EMD for the lines of interest. Since some Fe XIX line emissivities show modest density-sensitivity between the low density limit and densities expected under coronal conditions, we have used the APEC code to compute models for a wide range of densities. The most affected line ratio is that of $\lambda 101.55$ to $\lambda 108.37$. At $N_{e}=10^{10} \mathrm{~cm}^{-3}$ the predicted ratio is 0.347 (in photon units) compared with 0.261 at the standard APEC low density limit, in better agreement with the observed flux ratio of 0.328 .

Lack of significant variability further supports the assumption that the plasma conditions are stable, as individual lines of Fe XVII, Fe XVIII, and Fe XIX show modest flux

changes $(<10 \%$ deviation from the average value) between Chandra pointings and the 
lightcurves show low levels of variability $(\lesssim 8 \%)$ during a single pointing. There is also no evidence to challenge the standard assumptions of negligible optical depth (Canizares et al. 2000; Brown et al. 1998).

\section{Results and Discussion}

Figure 1 compares the observed Fe XVIII and Fe XIX line fluxes to those predicted by the spectral codes, APEC, CHIANTI V4.2 (Dere et al. 1997; Young et al. 2003), and SPEX V1.1 (Kaastra et al. 1996), which incorporates the MEKAL model (Mewe et al 1995). Emissivities provided by M. F. Gu (private communication, 2004) using the Flexible Atomic Code (FAC, Gu 2003) are also compared. The fluxes are scaled by the fluxes of their respective strong EUV resonance lines, for which direct excitation dominates. All models in the figure are calculated at a single temperature $T_{e}=6 \mathrm{MK}$, and the same density $N_{e}=10^{10}$ $\mathrm{cm}^{-3}$, except for SPEX, which is at the zero density limit.

Most striking is the discrepancy between the EUV and X-ray lines: the observed X-ray fluxes are stronger than predicted fluxes in all models. Even the X-ray $3 d-2 p$ resonance lines, Fe XVIII $\lambda 14.208$ and Fe XIX $\lambda 13.518$, are under-predicted relative to their EUV counterparts by more than $30 \%$ and a factor of 2 , respectively. Since these factors are larger than expected from calibration errors or line blending, it is possible that the accuracy of the direct excitation rate coefficients might explain the predicted weakness of $\lambda 14.208$ (see Brown et al. 2005); however, it is difficult to reconcile that with the larger discrepancy for $\lambda 13.518$.

The Fe XVIII and Fe XIX FUV forbidden line fluxes are in good agreement with the EUV line fluxes of $\lambda 93.92$ and $\lambda 108.37$ for the APEC models, and somewhat better than for the FAC rates. FAC does not calculate proton impact excitation rates, which are included 
in both APEC and CHIANTI. In APEC models, proton impact excitation increases the forbidden line emissivities by $15 \%$ and $8 \%$ for $\lambda 974.86$ and $\lambda 1118.07$, respectively. The predicted FUV line fluxes also begin to increase with density above $N_{e} \sim 10^{12} \mathrm{~cm}^{-3}$. APEC models give the best agreement at $N_{e}=2 \times 10^{12} \mathrm{~cm}^{-3}$, but are also consistent within observational errors with the lower coronal density range.

Figure 1 also shows some large discrepancies among the strongest X-ray lines, reflecting the $3 s-2 p / 3 d-2 p$ pattern. For these transitions the largest difference among the predictions results from the number of processes calculated with each model. For example, even though APEC and CHIANTI have similar collision strengths for the Fe XVIII $\lambda 15.625$ line, additional line flux in APEC is produced by direct excitation to $n=4$ and 5 levels, followed by radiative cascades, while CHIANTI currently includes only levels only up to $n=3$. On the other hand, for Fe XVIII $\lambda 16.07$ APEC and CHIANTI both show differences of more than a factor of 2 from FAC because neither includes the effects of DR on the upper level population, which are included in FAC.

Comparisons of APEC and FAC predictions to the observed fluxes of the X-ray lines listed in Table 1 are shown in Figure 2. We confirm a general $3 s-2 p / 3 d-2 p$ discrepancy pattern for APEC models that is largely removed with the FAC calculations. The $3 s-2 p / 3 d-$ $2 p$ ratios of the summed line fluxes from APEC are smaller than the observed ratios by $\sim 20 \%$, whereas FAC agreement is within $10 \%$. The inclusion of DR in the FAC models produces the additional $3 s-2 p$ line emissivity.

Another significant disagreement between the models and observations occurs for radiative transitions that terminate on excited levels, namely Fe XVIII $\lambda 15.870, \lambda 16.159$, and $\lambda 17.623$ and Fe XIX $\lambda 15.198$ and $\lambda 16.110$. Although the APEC line list, which is reasonably complete in this spectral region, does not include DR satellite lines from either Fe XVIII or Fe XIX, blending with satellite lines or lines from other ions cannot explain the extent of the 
under-prediction. It is possible that the large theoretical wavelength inaccuracies for these lines, up to a few percent, have led to misidentifications in the laboratory measurements. Blending of nearby lines from the same ion could produce such a pattern of under and overprediction. For Fe XVIII $\lambda 15.870$, this latter explanation is consistent with new wavelength calculations (Kotochigova et al. 2005; M. F. Gu 2005).

\section{Conclusions}

A surprising result of this benchmark spectral modeling study is the large discrepancy between modern theory and the Capella observations for the X-ray and EUV resonance lines of Fe XVIII (30\%) and Fe XIX (factor of 2). New FAC calculations including dielectronic recombination bring most X-ray lines into good agreement with observations; however puzzling discrepancies as large as a factor of 2 still remain for some relatively strong lines. Additional laboratory and theoretical work is needed to eliminate the largest remaining problems. Meanwhile, errors can largely be minimized by judicious choice of line diagnostics and consideration of appropriate atomic processes.

This work is supported in part by the Chandra X-ray Observatory Center (NAS8-39073).

We thank the CXC staff, particularly Harvey Tananbaum, for supporting efforts to obtain these data. We also acknowledge the efforts of the developers of the other public spectral modeling codes SPEX and CHIANTI as well as M. F. Gu for the atomic structure code FAC.

\section{REFERENCES}

Anders, E., Grevesse, N. 1989, GeCoA, 53, 197

Beiersdorfer, P., et al. 2002, ApJ, 576, L169 
Berrington, K. A., Seraph, H. E., \& Tully, J. A. 1998, A\&AS, 129, 161

Brickhouse, N. S. \& Drake, J. 2000, Revista Mexicana de Astronomia y Astrofisica Conf. Ser., 9,24

Brickhouse, N. S., Dupree, A. K., Edgar, R. J., Liedahl, D. A., Drake, S. A., White, N. E., \& Singh, K. P. 2000, ApJ, 530, 387

Brickhouse, N. S., Raymond, J. C., \& Smith, B. W. 1995, ApJS, 97, 551

Brinkman, A. C. et al. 2001, A\&A, 365, L324

Brown, G. V., Beiersdorfer, P., Liedahl, D. A., Widmann, K., \& Kahn, S. M. 1998, ApJ, 502,1015

Brown, G. V., Beiersdorfer, P., Liedahl, D. A., Widmann, K., Kahn, S. M., \& Clothiaux, E. J. 2002, ApJS, 140, 589

Brown, G. V. et al. 2005, in Proceedings of the Workshop on X-ray Diagnostics for Astrophysical Plasmas, ed. R. K. Smith, AIP, in press

Canizares, C. R. et al. 2000, ApJ, 539, L41

Cash, W. 1979, ApJ, 228, 939

Dere, K. P., Landi, E., Mason, H. E., Monsignori Fossi, B. C., \& Young, P. R. 1997, A\&A Supp. Ser., 125, 149

Dupree, A. K., Brickhouse N. S., Sanz-Forcada, J. 2005, ApJ, in preparation

Foster, V. J., Keenan, F. P., \& Reid, R. H. J. 1994, ADNDT, 58, 227

Freeman, P., Doe, S., Siemiginowska, A., 2001, SPIE, 4477, 76

Gu, M. F. 2003, ApJ, 582, 1241

Gu, M. F. 2005, ApJS, 156, 105 
Kaastra, J. S., Mewe, R., \& Nieuwenhuijzen, H. 1996, in Proceedings of the Eleventh Colloquium on UV and X-ray Spectroscopy of Laboratory and Astrophysical Plasmas, ed K. Yamashita and T. Watanabe, (Tokyo : Universal Academy Press), 411

Kimble, R. A., Davidsen, A. F., Long, K. S., \& Feldman, P. D. 1993, ApJ, 408, L41

Kotochigova, S., Kirby, K. P., Brickhouse, N. S., Mohr, P. J., \& Tupitsyn, I. I. 2005, in Proceedings of X-ray Diagnostics for Astrophysical Plasmas, AIP, ed. R. K. Smith, in press

Laming, J. M., et al. 2000, ApJ, 545, L161

Liedahl, D. A., Osterheld, A., \& Goldstein, W. 1995, ApJ, 438, L115

Mewe, R., Kaastra, J., \& Liedahl, D. A. 1995, Legacy, 6, 16

Piskunov, N., Wood, B. E., Linsky, J. L., Dempsey, R. C., \& Ayres, T. R. 1997, ApJ, 474, 315

Saba, J. L. R., Schmelz, J. T., Bhatia, A. K., \& Strong, K. T. 1999, ApJ, 510, 1064

Safranova, U. I., Vasilyev, A. A., \& Smith, R. K. 2001, Canadian J. Phys., 78, 1055

Smith, R. K., Brickhouse, N. S., Liedahl D. A., Raymond, J. A. 2001, ApJ, 556, L91

Xu, H., et al. 2002, ApJ, 579, 600

Young, P.,Dupree, A. K., Wood, B. E., Redfield, S., Linsky, J. L., Ake, T. B., Moos, H. W. 2001, ApJ, 555, L121

Young, P. R., Del Zanna, G., Landi, E., Dere, K. P., Mason, H. E., \& Landini, M. 2003, ApJS, 144, 135 


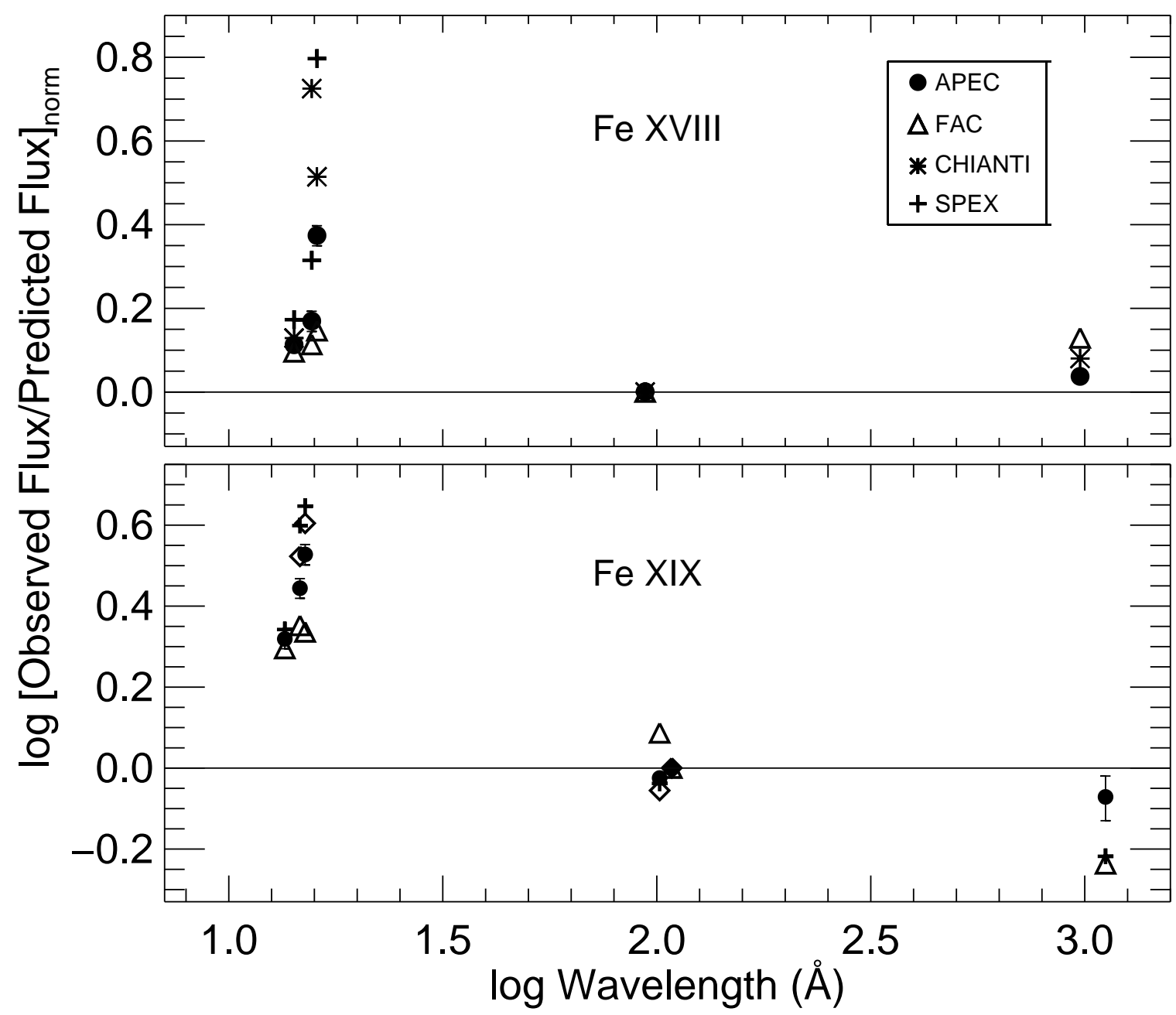

Fig. 1.- Observed to predicted flux ratios of strong lines in the X-ray, EUV, and FUV spectral regions. Shown for comparison are the ratios obtained using the APEC, CHIANTI, and SPEX spectral codes and the FAC rates. The density is $N_{e}=10.0 \mathrm{~cm}^{-3}$, except for SPEX. Top: Comparison for Fe XVIII lines, normalized to $\lambda$ 93.92. The X-ray lines plotted here are $\lambda 14.208, \lambda 15.625$, and $\lambda 16.071$. Bottom: Comparison for Fe XIX lines, normalized to $\lambda 108.37$. The X-ray lines plotted are $\lambda 13.518, \lambda 14.664$, and $\lambda 15.079$. 


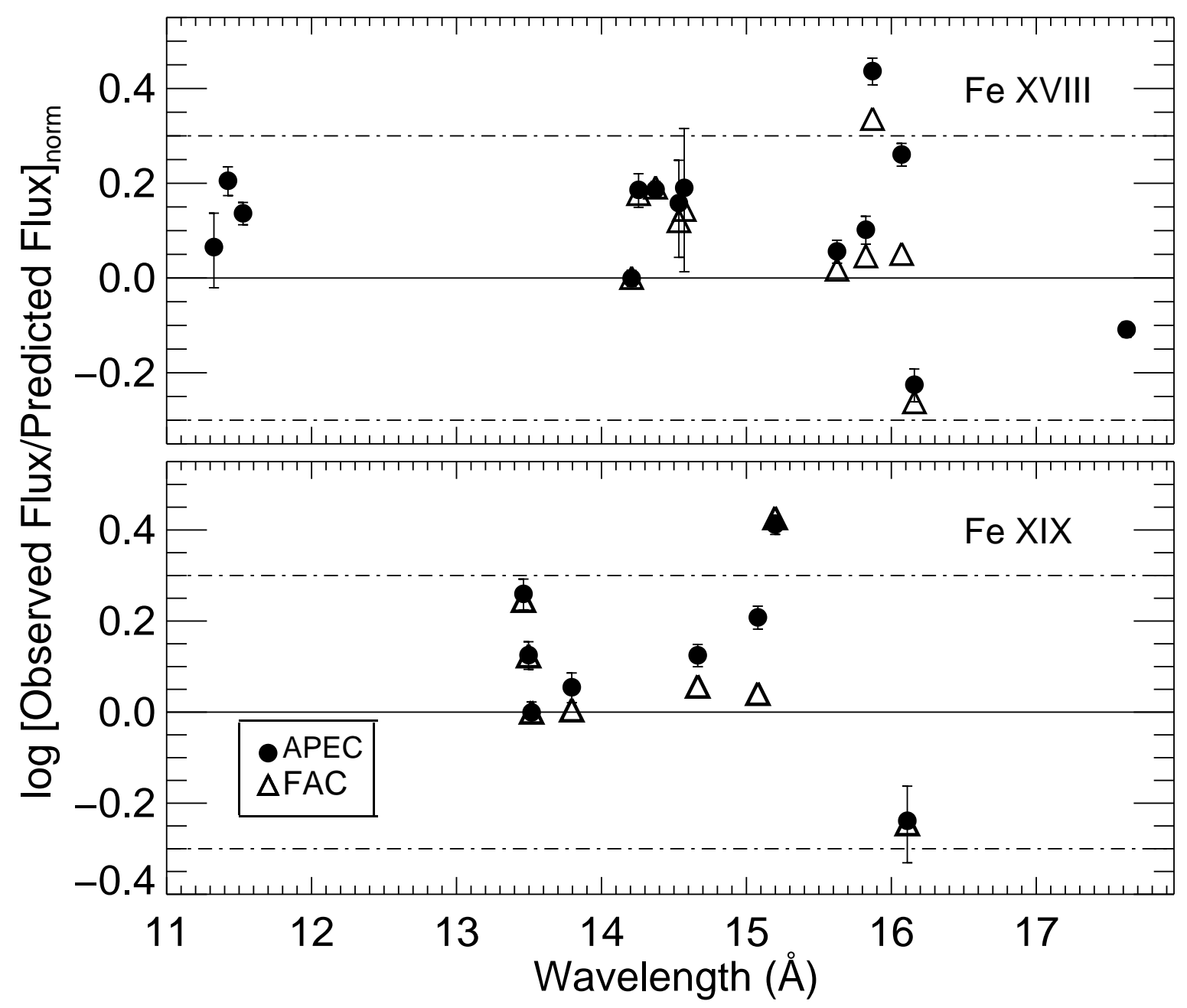

Fig. 2.- Observed to predicted flux ratios of X-ray lines using FAC and APEC. Lines from Table 1 excluding heavily blended Fe XVIII $\lambda 16.004$ are shown. Note the $3 d-2 p$ lines are between 14 and $15 \AA$ for Fe XVIII and shortward of $14 \AA$ for Fe XIX. Ratios are calculated at $N_{e}=10.0 \mathrm{~cm}^{-3}$. Dash-dotted lines represent agreement within a factor of 2. Top: Comparison for Fe XVIII, normalized to $\lambda 14.208$. There are no published FAC models for Fe XVIII $4 d-2 p$ lines around 11.4 A. Bottom: Comparison for Fe XIX, normalized to $\lambda 13.518$. 
Table 1. Fe XVIII and Fe XIX Line Measurements

\begin{tabular}{|c|c|c|c|c|c|c|c|}
\hline Inst & Ion & $\begin{array}{c}\lambda_{\text {ref }} \\
(\AA)\end{array}$ & $\begin{array}{c}\lambda_{o b s} \\
(\AA)\end{array}$ & Transition & $J_{U}-J_{L}$ & $\begin{array}{c}\text { Model Flux } \\
\left(\mathrm{ph} \mathrm{cm}^{-2} \mathrm{ks}^{-1}\right)\end{array}$ & $\begin{array}{c}\text { Observed Flux } \\
\left(\mathrm{ph} \mathrm{cm}^{-2} \mathrm{ks}^{-1}\right)\end{array}$ \\
\hline FUSE $^{\mathrm{b}}$ & Fe XVIII & 974.86 & 974.85 & $2 p^{5}{ }^{2} P_{1 / 2}-2 p^{5}{ }^{2} P_{3 / 2}$ & $\frac{1}{2}-\frac{3}{2}$ & 5.063 & $5.50 \pm 0.03$ \\
\hline LETG & Fe XVIII & 103.93 & 103.98 & $2 s 2 p^{6}{ }^{2} S_{1 / 2}-2 p^{5}{ }^{2} P_{1 / 2}$ & $\frac{1}{2}-\frac{1}{2}$ & 1.625 & $1.69 \pm 0.05$ \\
\hline LETG & Fe XVIII & 93.923 & 94.02 & $2 s 2 p^{6}{ }^{2} S_{1 / 2}-2 p^{5}{ }^{2} P_{3 / 2}$ & $\frac{1}{2}-\frac{3}{2}$ & 4.441 & $4.44 \pm 0.03$ \\
\hline MEG & Fe XVIII & 17.623 & 17.620 & $2 p^{4} 3 p^{2} P_{3 / 2}-2 s 2 p^{6}{ }^{2} S_{1 / 2}$ & $\frac{3}{2}-\frac{1}{2}$ & 0.300 & $0.30 \pm 0.01$ \\
\hline MEG & Fe XVIII & 16.159 & 16.163 & $2 s 2 p^{5} 3 s^{2} P_{3 / 2}-2 s 2 p^{6}{ }^{2} S_{1 / 2}$ & $\frac{3}{2}-\frac{1}{2}$ & 0.164 & $0.13 \pm 0.00$ \\
\hline $\mathrm{MEG}^{\mathrm{c}}$ & Fe XVIII & 16.071 & 16.073 & $2 p^{4}\left({ }^{3} P\right) 3 s^{4} P_{5 / 2}-2 p^{5}{ }^{2} P_{3 / 2}$ & $\frac{5}{2}-\frac{3}{2}$ & 0.418 & $0.82 \pm 0.01$ \\
\hline $\mathrm{HEG}^{\mathrm{c}}$ & Fe XVIII & 16.071 & 16.076 & $2 p^{4}\left({ }^{3} P\right) 3 s{ }^{4} P_{5 / 2}-2 p^{5}{ }^{2} P_{3 / 2}$ & $\frac{5}{2}-\frac{3}{2}$ & 0.418 & $1.00 \pm 0.06$ \\
\hline HEG & Fe XVIII ${ }^{d}$ & 16.004 & 16.008 & $2 p^{4}\left({ }^{3} P\right) 3 s^{2} P_{3 / 2}-2 p^{5}{ }^{2} P_{3 / 2}$ & $\frac{3}{2}-\frac{3}{2}$ & 0.768 & $0.81 \pm 0.04$ \\
\hline HEG & Fe XVIII & 15.870 & 15.873 & $2 p^{4}\left({ }^{1} D\right) 3 s^{2} D_{3 / 2}-2 p^{5}{ }^{2} P_{1 / 2}$ & $\frac{3}{2}-\frac{1}{2}$ & 0.095 & $0.34 \pm 0.02$ \\
\hline HEG & Fe XVIII & 15.824 & 15.831 & $2 p^{4}\left({ }^{3} P\right) 3 s{ }^{4} P_{3 / 2}-2 p^{5}{ }^{2} P_{3 / 2}$ & $\frac{3}{2}-\frac{3}{2}$ & 0.179 & $0.29 \pm 0.02$ \\
\hline HEG & Fe XVIII & 15.625 & 15.628 & $2 p^{4}\left({ }^{1} D\right) 3 s^{2} D_{5 / 2}-2 p^{5}{ }^{2} P_{3 / 2}$ & $\frac{5}{2}-\frac{3}{2}$ & 0.290 & $0.43 \pm 0.02$ \\
\hline HEG & Fe XVIII & 14.571 & 14.559 & $2 p^{4}\left({ }^{3} P\right) 3 d{ }^{4} P_{3 / 2}-2 p^{5}{ }^{2} P_{3 / 2}$ & $\frac{3}{2}-\frac{3}{2}$ & 0.110 & $0.21 \pm 0.07$ \\
\hline HEG & Fe XVIII & 14.534 & 14.539 & $2 p^{4}\left({ }^{3} P\right) 3 d^{2} F_{5 / 2}-2 p^{5}{ }^{2} P_{3 / 2}$ & $\frac{5}{2}-\frac{3}{2}$ & 0.210 & $0.39 \pm 0.09$ \\
\hline HEG & Fe XVIII & 14.373 & 14.376 & $2 p^{4}\left({ }^{3} P\right) 3 d^{2} D_{5 / 2}-2 p^{5}{ }^{2} P_{3 / 2}$ & $\frac{5}{2}-\frac{3}{2}$ & 0.278 & $0.55 \pm 0.02$ \\
\hline HEG & $\mathrm{Fe} X V I I I^{\mathrm{e}}$ & 14.256 & 14.261 & $2 p^{4}\left({ }^{1} D\right) 3 d^{2} S_{1 / 2}-2 p^{5}{ }^{2} P_{3 / 2}$ & $\frac{1}{2}-\frac{3}{2}$ & 0.087 & $0.42 \pm 0.03$ \\
\hline$\ldots$ & $\ldots$ & $\ldots$ & $\ldots$ & $2 p_{1 / 2} 2 p_{3 / 2}^{3} 3 d_{5 / 2}-2 p^{5}{ }^{2} P_{3 / 2}$ & $\frac{5}{2}-\frac{3}{2}$ & 0.141 & $\ldots$ \\
\hline HEG & Fe XVIII & 14.208 & 14.208 & $2 p_{1 / 2} 2 p_{3 / 2}^{3} 3 d_{5 / 2}-2 p^{5}{ }^{2} P_{3 / 2}$ & $\frac{3}{2}-\frac{3}{2}$ & 0.381 & $1.40 \pm 0.05$ \\
\hline$\ldots$ & $\ldots$ & $\ldots$ & $\cdots$ & $2 p^{4}\left({ }^{1} D\right) 3 d^{2} D_{5 / 2}-2 p^{5}{ }^{2} P_{3 / 2}$ & $\frac{5}{2}-\frac{3}{2}$ & 0.695 & $\cdots$ \\
\hline HEG & Fe XVIII & 11.527 & 11.528 & $2 p_{1 / 2}^{2} 2 p_{3 / 2}^{2} 4 d_{5 / 2}-2 p^{5}{ }^{2} P_{3 / 2}$ & $\frac{5}{2}-\frac{3}{2}$ & 0.032 & $0.17 \pm 0.01$ \\
\hline$\ldots$ & $\ldots$ & $\ldots$ & $\ldots$ & $2 p^{4}\left({ }^{3} P\right) 4 d^{2} D_{5 / 2}-2 p^{5}{ }^{2} P_{3 / 2}$ & $\frac{5}{2}-\frac{3}{2}$ & 0.061 & $\ldots$ \\
\hline HEG & Fe XVIII & 11.423 & 11.424 & $2 p^{4}\left({ }^{3} P\right) 4 d^{2} F_{5 / 2}-2 p^{5}{ }^{2} P_{3 / 2}$ & $\frac{5}{2}-\frac{3}{2}$ & 0.080 & $0.13 \pm 0.01$ \\
\hline$\ldots$ & Fe XXII & 11.427 & $\cdots$ & $2 s 2 p_{1 / 2} 3 p_{3 / 2}-2 p^{2} P_{1 / 2}$ & $\frac{3}{2}-\frac{1}{2}$ & 0.007 & $\ldots$ \\
\hline HEG & Fe XVIII & 11.326 & 11.327 & $2 p^{4}\left({ }^{1} D\right) 4 d^{2} S_{1 / 2}-2 p^{5}{ }^{2} P_{3 / 2}$ & $\frac{1}{2}-\frac{3}{2}$ & 0.019 & $0.13 \pm 0.02$ \\
\hline$\cdots$ & $\cdots$ & $\ldots$ & $\cdots$ & $2 p^{4}\left({ }^{1} D\right) 4 d^{2} P_{3 / 2}-2 p^{5}{ }^{2} P_{3 / 2}$ & $\frac{3}{2}-\frac{3}{2}$ & 0.031 & $\ldots$ \\
\hline$\ldots$ & $\cdots$ & $\ldots$ & $\cdots$ & $2 p^{4}\left({ }^{1} D\right) 4 d^{2} D_{5 / 2}-2 p^{5}{ }^{2} P_{3 / 2}$ & $\frac{5}{2}-\frac{3}{2}$ & 0.038 & $\cdots$ \\
\hline FUSE & Fe $\mathrm{XIX}^{\mathrm{b}, f}$ & 1118.07 & $\cdots$ & $2 p^{4}{ }^{3} P_{1}-2 p^{4}{ }^{3} P_{2}$ & $1-2$ & 1.833 & $1.74 \pm 0.22$ \\
\hline LETG & Fe XIX & 120.00 & 120.04 & $2 s 2 p^{5}{ }^{3} P_{2}-2 p^{4}{ }^{3} P_{1}$ & $2-1$ & 0.836 & $0.97 \pm 0.03$ \\
\hline LETG & Fe XIX & 111.70 & 111.74 & $2 s 2 p^{5}{ }^{3} P_{1}-2 p^{4}{ }^{3} P_{1}$ & $1-1$ & 0.326 & $0.46 \pm 0.02$ \\
\hline LETG & Fe XIX & 109.97 & 109.99 & $2 s 2 p^{5}{ }^{3} P_{1}-2 p^{4}{ }^{3} P_{0}$ & $1-0$ & 0.413 & $0.46 \pm 0.02$ \\
\hline LETG & Fe XIX & 108.37 & 108.39 & $2 s 2 p^{5}{ }^{3} P_{2}-2 p^{4}{ }^{3} P_{2}$ & $2-2$ & 3.091 & $3.13 \pm 0.05$ \\
\hline LETG & Fe XIX & 101.55 & 101.59 & $2 s 2 p^{5}{ }^{3} P_{1}-2 p^{4}{ }^{3} P_{2}$ & $1-2$ & 0.838 & $1.02 \pm 0.03$ \\
\hline LETG & Fe XIX & 91.02 & 91.054 & $2 s 2 p^{5}{ }^{1} P_{1}-2 p^{4}{ }^{1} D_{2}$ & $1-2$ & 0.241 & $0.45 \pm 0.02$ \\
\hline HEG & Fe XIX & 16.110 & 16.111 & $2 p_{1 / 2} 2 p_{3 / 2}^{2} 3 p_{1 / 2}-2 s 2 p^{5}{ }^{3} P_{2}$ & $2-2$ & 0.120 & $0.14 \pm 0.03$ \\
\hline HEG & Fe XIX & 15.198 & 15.204 & $2 p_{1 / 2}^{2} 2 p_{3 / 2}^{2} 3 s-2 s 2 p^{5}{ }^{3} P_{2}$ & $2-2$ & 0.080 & $0.39 \pm 0.02$ \\
\hline HEG & Fe XIX & 15.079 & 15.083 & $2 p^{3}\left({ }^{4} S\right) 3 s{ }^{5} S_{2}-2 p^{4}{ }^{3} P_{2}$ & $2-2$ & 0.094 & $0.33 \pm 0.02$ \\
\hline HEG & Fe XIX & 14.664 & 14.671 & $2 p^{3}\left({ }^{2} D\right) 3 s{ }^{3} D_{3}-2 p^{4}{ }^{3} P_{2}$ & $3-2$ & 0.079 & $0.21 \pm 0.01$ \\
\hline HEG & Fe XIX & 13.795 & 13.795 & $2 p_{1 / 2} 2 p_{3 / 2}^{2} 3 d_{5 / 2}-2 p^{4}{ }^{3} P_{2}$ & $3-2$ & 0.105 & $0.24 \pm 0.02$ \\
\hline$\cdots$ & $\cdots$ & $\ldots$ & $\cdots$ & $2 p^{3}\left({ }^{2} D\right) 3 d^{3} P_{2}-2 p^{4}{ }^{3} P_{2}$ & $3-2$ & 0.012 & $\cdots$ \\
\hline HEG & Fe XIX & 13.518 & 13.523 & $2 p^{3}\left({ }^{2} D\right) 3 d{ }^{3} D_{3}-2 p^{4}{ }^{3} P_{2}$ & $3-2$ & 0.262 & $0.52 \pm 0.03$ \\
\hline HEG & Fe XIX & 13.497 & 13.507 & $2 p_{1 / 2} 2 p_{3 / 2}^{2} 3 d_{3 / 2}-2 p^{4}{ }^{3} P_{2}$ & $2-2$ & 0.118 & $0.32 \pm 0.02$ \\
\hline$\cdots$ & $\mathrm{Fe} X \mathrm{XI}$ & 13.507 & 13.507 & $1 s^{2} 2 s 2 p_{1 / 2}^{2} 3 s-1 s^{2} 2 s 2 p^{3}{ }^{3} D_{1}$ & $2-2$ & 0.025 & $\ldots$ \\
\hline HEG & Fe XIX & 13.462 & 13.470 & $2 p^{3}\left({ }^{2} D\right) 3 d^{3} S_{1}-2 p^{4}{ }^{3} P_{2}$ & $1-2$ & 0.072 & $0.25 \pm 0.02$ \\
\hline HEG & Ne IX & 13.447 & 13.446 & $1 s^{2}{ }^{1} S_{0}-1 s 2 p^{1} P_{1}$ & $1-2$ & 0.397 & $0.40 \pm 0.02$ \\
\hline
\end{tabular}


${ }^{a}$ Line blends are listed separately if they contribute $>10 \%$ to the Fe line of interest (in the model). Fluxes (including blends) normalized to the $\lambda 93.92$ line predicted by the emission measure distribution model using APEC at a density of $1 \mathrm{~cm}^{-3}$ are listed. The observed fluxes have been corrected for interstellar absorption using $N_{H}=1.7 \times 10^{18} \mathrm{~cm}^{-2}$ (Piskunov 1997), neutral helium, and $\mathrm{H} / \mathrm{He}$ abundance ratio set at 11.6 (Kimble et al. 1993). The largest correction at $\lambda 120.0$ amounts to only $9 \%$.

b See Young et al. (2001).

c MEG and HEG measurements of this line are given to show the cross-calibration. HEG is preferred for this analysis because of its better spectral resolution.

d Contribution of OVIII to this line is more than $50 \%$.

e LETG flux was measured to cross check the calibration of LETG vs HETG. The LETG line is somewhat blended, but the flux is within $30 \%$ of the HETG flux.

$\mathrm{f}$ This FUSE measurement is uncertain as this line is blended. Solar network spectra were used to estimate the contribution of $\mathrm{C}$ to the blend. 